\title{
Percepção de controle e qualidade de vida: comparação entre idosos institucionalizados e não institucionalizados
}

\section{Perceived control and quality of life: comparison between elderly living in nursing homes and in the community}

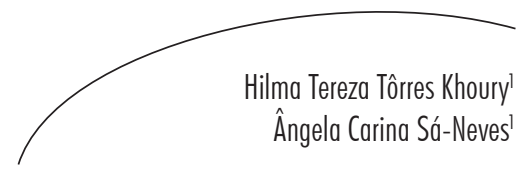

\section{Resumo}

Introdução: Percepção de controle é um recurso psicossocial associado à qualidade de vida e bem-estar. Qualidade de vida é um conceito subjetivo e multidimensional. Percepção de controle refere-se à crença de estar no comando da própria vida e dos eventos a ela pertinentes. Idosos institucionalizados geralmente estão sujeitos a inúmeras restrições que podem afetar sua percepção de controle e qualidade de vida. Objetivo: Investigar percepções de controle - primário e secundário - e qualidade de vida em uma amostra de idosos em Belém-PA, comparando-se institucionalizados com residentes na comunidade, buscando-se associação entre estas variáveis nesses contextos de existência. Método: Trata-se de estudo analítico, de observação, com corte transversal. Foram avaliadas 66 pessoas entre 60 e 96 anos (21 homens; 45 mulheres; 33 institucionalizados; 33 da comunidade), por meio dos instrumentos WHOQOL-OLD e ECOPSE. Resultados: Diferenças significativas $(\mathrm{p} \leq 0,05)$ entre os idosos institucionalizados e os que viviam na comunidade foram encontradas. A percepção de estar no controle aparece associada a satisfação com conquistas na vida e anseios em ambos os grupos, contudo, a associação entre controle e autonomia é encontrada apenas nos residentes da comunidade. Conclusão: Viver na comunidade favorece o exercício do controle e a qualidade de vida; a percepção de controle está associada à qualidade de vida em maior número de dimensões nos idosos que vivem na comunidade, comparados aos institucionalizados.

\section{Abstract}

Introduction: Perceived control is a psychosocial resource, which has been associated to quality of life and well-being. Quality of life is a subjective and multidimensional construct. Perceived control refers to beliefs of managing own life. Elderly living in nursing homes are generally subject to numerous restrictions that can affect their

Faculdade de Psicologia, Instituto de Filosofia e Ciências Humanas. Universidade Federal do Pará. Belém, PA, Brasil.

Os dados analisados neste artigo são um recorte do projeto "Velhice bem-sucedida: fatores psicossociais associados à velhice saudável e qualidade de vida", coordenado pela primeira autora. Tal recorte compôs o plano de trabalho da segunda autora quando bolsista de iniciação cientifica - PIBIC/UFPA, sob orientação da primeira autora.

Palavras-chave: Idoso. Instituição de Longa Permanência para Idosos. Controle Percebido. Qualidade de Vida. 
perceived control and quality of life. Objective: To investigate perceptions of control - primary and secondary - and quality of life in elderly, in the city of Belém-PA, comparing the institutionalized ones with those living in the community, looking for association between these variables in these contexts of existence. This is an analytical study, observational, cross-sectional, in which 66 people aged between 60 and 96 years ( 21 men, 45 women; 33 institutionalized; 33 living in the community) were assessed through the instruments WHOQOL-OLD and ECOPSE. Results: Significant differences $(\mathrm{p} \leq 0,05)$ between institutionalized elderly and the ones living in the community were found. Beliefs of being in control are associated to satisfaction with achievements and interests in both groups; however, the association between control and autonomy is found only among community residents. Conclusion: Living in the community favors perceived control and quality of life; perceived control is associated with quality of life in a great number of dimensions in the elderly living in the community, compared to institutionalized ones.
Key words: Elderly. Homes for the Aged. Perceived Control. Quality of Life.

\section{INTRODUÇÃO}

A qualidade de vida $(\mathrm{QV})$ na velhice tem sido motivo de amplas discussões em todo o mundo, com foco em preservação da saúde e bem-estar global nessa fase da vida., ${ }^{1,2}$ QV é um construto complexo e multidimensional que requer abordagem de diferentes ângulos, levando-se em consideração aspectos subjetivos e objetivos ${ }^{3,4}$ e dimensões positivas e negativas. ${ }^{4}$ Com base nesta perspectiva, o grupo de qualidade de vida da Organização Mundial da Saúde (OMS) definiu qualidade de vida como a "percepção do indivíduo de sua posição na vida, no contexto da cultura e sistema de valores nos quais ele vive, e em relação aos seus objetivos, expectativas, padrões e preocupações". ${ }^{4}$

A qualidade de vida na velhice é fortemente determinada pela manutenção da capacidade funcional - independência e autonomia. ${ }^{5,6}$ Autonomia se caracteriza pela habilidade de tomar decisões e controlar a própria vida. Independência é a capacidade para executar as atividades de vida diária.?

Manter a capacidade funcional - critério atual de velhice saudável ${ }^{6}$ - não depende apenas de fatores orgânicos. Fatores psicológicos, como as crenças de autoeficácia ${ }^{8}$ e a percepção de controle, ${ }^{9-15}$ desempenham papel fundamental na manutenção da saúde e independência na velhice e estão associadas a qualidade de vida, bem-estar ou satisfação com a vida.

A percepção de controle ou controle percebido aparece na literatura da área sob diferentes denominações, tais como senso de controle ${ }^{12}$ e lócus de controle, ${ }^{14}$ mas o sentido é o mesmo. Diz respeito a crenças pessoais acerca da própria capacidade para controlar eventos e ameaças. ${ }^{16}$ Rothbaum, Weisz \& Snyder ${ }^{17}$ classificaram a percepção de controle como controle primário (CP) e controle secundário (CS). O CP refere-se a esforços para dominar o ambiente - físico/social - e adaptá-lo às próprias necessidades. O CS diz respeito a esforços para se adaptar ao ambiente, especialmente a situações em que metas não foram alcançadas ou ocorreram perdas/falhas. ${ }^{10,18}$

No presente trabalho, adotou-se a categorização obtida em estudo realizado no Brasil $^{10,18}$ e que avaliou esses construtos da seguinte forma: 1) Controle primário (CP), em duas categorias: a) esforço de realização com os próprios recursos do indivíduo (CP1); b) esforço de realização com ajuda - de outras pessoas ou de tecnologias (CP2), visando atingir metas; 2) Controle secundário (CS), avaliado em uma única categoria, sendo considerado como esforços de adaptação. Em ambos, CP e CS, esses esforços podem ser em nível de ação/comportamento ou de cognição/pensamento. 
A literatura evidencia relação entre percepção de controle e indicadores de qualidade de vida em pessoas idosas que vivem na comunidade. Lachman \& Agrigoroaei ${ }^{15}$ conduziram estudo longitudinal com adultos e idosos, concluindo que a manutenção da saúde funcional está associada a fatores protetores de natureza psicológica e social, entre os quais as crenças de controle. Ferguson \& Goodwin ${ }^{19}$ investigaram idosos em estudo transversal, buscando associação entre recursos psicossociais e bem-estar, tendo concluído que a percepção de controle atua como mediadora da relação entre otimismo e bemestar psicológico. Bowling et al. ${ }^{20}$ estudaram idosos com funcionamento físico prejudicado, mas que avaliaram sua qualidade de vida como boa, comparando-os com aqueles que a avaliaram como ruim. A distinção entre eles se deveu a fatores tais como melhor saúde autoavaliada, menor carga de doenças crônicas, não ter caído, maior engajamento social e níveis mais elevados de percepção de controle sobre a vida. Haynes et al. ${ }^{13}$ conduziram estudo com pessoas muito idosas (79-98 anos), buscando relação entre a percepção de controle - primário e secundário - e bemestar físico e psicológico. Aqueles que utilizavam estratégias de controle primário (persistência nas tarefas) ou estratégias combinadas de controle primário e controle secundário (compensação) para lidar com as restrições da velhice relataram melhor bem-estar físico e psicológico, comparados com os que abandonaram as estratégias de controle primário ou utilizavam mais estratégias de controle secundário.

Pesquisas empíricas realizadas no Brasil também encontraram relação entre percepção de controle e qualidade de vida em idosos da comunidade. Investigação realizada na capital do Brasil com 315 idosos (60-92 anos) revelou associação entre percepção de controle primário e diversos indicadores de qualidade de vida - como independência, estado de saúde, amplas redes de relações sociais e engajamento em diversas atividades, entre outros. Em Minas Gerais, investigação ${ }^{21}$ com 360 idosos demonstrou associação entre senso de controle e qualidade de vida. No Rio Grande do Sul, estudo ${ }^{22}$ sobre qualidade de vida e lócus de controle em idosos mostrou melhor qualidade de vida entre aqueles com lócus de controle interno (que atribuíam a si próprios a causa dos eventos que lhes sucediam) e que possuíam uma vida mais ativa.

Os dados apresentados atestam que perceberse no controle contribui para a qualidade de vida de idosos que residem na comunidade. Perguntase, entretanto, como se apresentaria a relação entre percepção de controle e qualidade de vida em idosos institucionalizados? As instituições de longa permanência para idosos (ILPIs) são atualmente uma das alternativas de cuidados não familiares diante do contexto de grandes mudanças em que se insere o envelhecimento populacional. $^{23}$ Fatores como mudanças na configuração familiar, devido à transformação nos papéis sociais desempenhados pelas mulheres, tradicionais cuidadoras, bem como alterações nas uniões matrimoniais e redução das taxas de fecundidade e mortalidade têm contribuído para a institucionalização. ${ }^{23}$

Contudo, o ambiente oferecido pelas ILPIs pode favorecer ou dificultar o exercício da autonomia e, consequentemente, a percepção de controle. Ambientes que oferecem tranquilidade, conforto, privacidade, oportunidade para relações sociais, atividades variadas no dia a dia e atividades externas são favoráveis. Quando estas condições estão ausentes, o ambiente costuma ser percebido como arregimentado, restrito, rotineiro e enfadonho. ${ }^{24}$ No Brasil, mais de $65 \%$ das ILPIs são de natureza filantrópica, ${ }^{23} \mathrm{o}$ que significa que a maioria possui poucos recursos financeiros que lhes permitam oferecer um ambiente rico e variado, restringindo assim as possibilidades de o idoso exercer o autogoverno e o poder de decisão - portanto, controle.

Esse tipo de contexto institucional poderia afetar negativamente a qualidade de vida dos idosos abrigados. Assim, perguntase, como se apresentam as percepções de controle e de qualidade de vida entre idosos institucionalizados? Haveria relação entre 
percepção de controle e qualidade de vida no contexto institucional? Haveria diferença com relação a estas questões, comparando-se com idosos que vivem na comunidade?

Estudos relacionando percepção de controle e qualidade de vida no contexto institucional com população idosa não foram encontrados nas bases de dados disponíveis. Há diversos estudos, anteriormente citados, mostrando associação entre estas duas variáveis, mas com idosos que vivem na comunidade. A literatura mostra alguns estudos acerca da qualidade de vida entre idosos institucionalizados, ${ }^{25-27}$ bem como estudos que comparam idosos que vivem na comunidade com institucionalizados, ${ }^{28-30}$ mas sem fazer relação com percepção de controle.

Entre os estudos que avaliaram qualidade de vida em idosos institucionalizados no Brasil utilizando o instrumento do grupo de qualidade de vida da OMS para idosos (WHOQOLOLD), ${ }^{26,27,30}$ o índice global de qualidade de vida foi moderado em dois deles. ${ }^{26,27} \mathrm{~A}$ faceta "autonomia" foi a que obteve menor pontuação; as dimensões que alcançaram maior pontuação foram "Morte e Morrer"26 e "Funcionamento do Sensório". 27,30

Dos estudos que compararam idosos institucionalizados e residentes na comunidade com relação à qualidade de vida, ${ }^{28-30}$ dois utilizaram o WHOQOLbref ${ }^{28,29}$ (não específico para população idosa), tendo encontrado diferenças significativas a favor dos residentes na comunidade. Um dos estudos ${ }^{30}$ acessou qualidade de vida tanto por meio do WHOQOLbref quanto do WHOQOL-OLD e não encontrou diferença significativa comparando idosos dos dois contextos.

Diante do exposto, objetivou-se investigar as percepções de controle (primário e secundário) e de qualidade de vida em uma amostra de idosos na cidade de Belém-PA, comparandose institucionalizados com residentes na comunidade, buscando-se associação entre estas variáveis nesses contextos de existência.

\section{MÉTODO}

Trata-se de estudo analítico, de observação, com corte transversal, realizado com idosos que viviam na comunidade e residentes em ILPIs, para fins de comparação, no período de março a junho de 2010.

\section{Participantes e contextos}

Participaram 66 idosos (21 homens; 45 mulheres), sendo 33 vivendo em ILPIs, e 33 na comunidade, com idades entre 60 e 96 anos $(\mathrm{m}=74,9 ; \mathrm{dp}=8,89)$. Os idosos da comunidade eram independentes e foram contatados em um Centro de Atenção à Saúde do Idoso. Os idosos institucionalizados foram procurados em duas ILPIs de Belém-PA, sendo uma pública e outra privada. Alguns deles eram independentes e outros parcialmente dependentes para algumas atividades básicas de vida diária, segundo informações obtidas com profissionais (assistentes sociais e cuidadores) da instituição. A seleção levou em conta os seguintes critérios de inclusão: ter 60 anos de idade ou mais, demonstrar, durante o contato inicial com as pesquisadoras, condição cognitiva para responder aos questionários e aceitar fazer parte da pesquisa.

Instrumentos

Os instrumentos de medida utilizados foram: (a) questionário sociodemográfico, que incluía informações sobre idade, sexo, renda, nível de escolaridade e ocupação, entre outras; (b) questionário para avaliar qualidade de vida WHOQOL-OLD, da OMS ${ }^{31}$ - contendo 24 itens avaliados em escala do tipo Likert com cinco pontos. Os itens medem seis facetas ou dimensões da qualidade de vida: Funcionamento do Sensório (FS), Autonomia (AUT), Atividades Passadas, Presentes e Futuras (PPF), Participação Social (PSO), Morte e Morrer (MEM) e Intimidade (INT) (quadro 1); (c) questionário para aferir percepção de controle, primário e secundário - 
ECOPSE, ${ }^{10}$ composto por 20 itens avaliados em escala de cinco pontos, tipo Likert $(1=$ nada a ver comigo; $5=$ tudo a ver comigo). Os itens medem três fatores: controle primário com recursos próprios (CP1), controle primário com ajuda (CP2) e controle secundário (CS) (quadro 2).

Quadro 1. Descrição das dimensões componentes do WHOQOL-OLD. ${ }^{31}$

\begin{tabular}{|l|l|l|}
\hline \multicolumn{1}{|c|}{ Sigla } & \multicolumn{1}{|c|}{ Nome da dimensão } & \multicolumn{1}{c|}{ Definição } \\
\hline FS & Funcionamento do sensório & $\begin{array}{l}\text { Avalia o funcionamento sensorial e o impacto da perda } \\
\text { das habilidades sensoriais na qualidade vida. }\end{array}$ \\
\hline AUT & Autonomia & $\begin{array}{l}\text { Descreve até que ponto se é capaz de viver de forma } \\
\text { autônoma e tomar as próprias decisões. }\end{array}$ \\
\hline PPF & Atividades passadas, presentes e futuras & $\begin{array}{l}\text { Refere-se à satisfação sobre conquistas na vida e coisas } \\
\text { a que se anseia. }\end{array}$ \\
\hline PSO & Participação social & $\begin{array}{l}\text { Delineia a participação em atividades do cotidiano, } \\
\text { especialmente na comunidade. }\end{array}$ \\
\hline MEM & Morte e morrer & $\begin{array}{l}\text { Relaciona-se a preocupações, inquietações e temores } \\
\text { sobre a morte e o morrer. }\end{array}$ \\
\hline INT & Intimidade & $\begin{array}{l}\text { Avalia a percepção de sentir-se amado e apoiado, assim } \\
\text { como de amar. }\end{array}$ \\
\hline
\end{tabular}

Quadro 2. Descrição das dimensões componentes da ECOPSE. ${ }^{10}$

\begin{tabular}{|l|l|l|}
\hline Sigla & \multicolumn{1}{|c|}{ Nome da dimensão } & \multicolumn{1}{c|}{ Definição } \\
\hline CP1 & Controle primário com recursos próprios & $\begin{array}{l}\text { Diz respeito a esforços de realização dirigidos à } \\
\text { concretização de metas, por meio dos próprios } \\
\text { recursos pessoais do indivíduo. }\end{array}$ \\
\hline CP2 & Controle primário com ajuda & $\begin{array}{l}\text { Concernente a esforços de realização dirigidos à } \\
\text { concretização de metas, porém com ajuda seja de } \\
\text { outras pessoas ou de tecnologias. }\end{array}$ \\
\hline CS & Controle secundário & $\begin{array}{l}\text { Refere-se a esforços de adaptação (compensação ou } \\
\text { consolo) em caso de metas não alcançadas ou difíceis } \\
\text { de alcançar; perdas ou falhas. }\end{array}$ \\
\hline
\end{tabular}




\section{Procedimentos}

Após aprovação da pesquisa pelo Comitê de Ética em Pesquisa em seres humanos, do Instituto de Ciências da Saúde da Universidade Federal do Pará, sob no de protocolo 009/09, buscouse o consentimento da direção das instituições/ organizações envolvidas na realização da pesquisa - ILPIs e Centro de Atenção a Saúde do Idoso, acima mencionados.

Em ambos os contextos - ILPIs e comunidade -, os idosos foram selecionados de forma não probabilística, conforme os critérios de elegibilidade descritos na seção de participantes. No Centro de Atenção à Saúde do Idoso, os potenciais participantes foram abordados enquanto aguardavam consulta ou quando haviam terminado alguma atividade de grupo. Nas ILPIs, a abordagem era realizada em horários que não coincidiam com os das refeições ou descanso, geralmente quando o idoso estava em áreas de estar ou lazer - por exemplo, na sala de TV e na maloca (barraca de madeira com bancos, em formato redondo, situada no meio do jardim, onde costumam conversar ou realizar atividades de grupo).

Em ambos os contextos, caso aceitassem participar, o Termo de Consentimento Livre e Esclarecido era lido e assinado e, consequentemente, os questionários eram aplicados na seguinte ordem: questionário sociodemográfico, WHOQOL-OLD e ECOPSE. Os dados foram coletados em sessão única, com duração aproximada de 40 minutos.
Ao final, agradecia-se a participação ofertandose um informativo sobre as Leis de Amparo ao Idoso e Saúde na Velhice.

Análise dos dados

As análises foram efetuadas por meio de testes não paramétricos. A comparação entre os idosos residentes nas ILPIs e os que viviam na comunidade, com relação às percepções de controle (primário e secundário) e de qualidade de vida, foi realizada por meio do teste MannWhitney. As correlações entre percepção de controle - primário e secundário - e qualidade de vida foram testadas pelo coeficiente de correlação de Spearman e foram realizadas separadamente por grupo. Para todas as provas, foi adotado o valor de $\mathrm{p} \leq 0,05$.

\section{RESULTADOS}

Com relação à caracterização sociodemográfica, destaca-se que os residentes das ILPIs, comparados aos que viviam na comunidade, eram mais velhos, possuíam escolaridade e renda individual mais baixas, tinham menos amigos, raramente ou nunca recebiam visitas e não tinham um quarto só para si. Cabe destacar que mais de 30\% dos idosos das ILPIs eram analfabetos e tinham renda individual quase duas vezes menor que os idosos que viviam na comunidade. Contudo, apesar disso, $12,1 \%$ dos residentes nas ILPIs possuíam dependentes econômicos (tabela 1). 
Tabela 1. Comparação entre idosos residentes na comunidade e em ILPIs - variáveis sociodemográficas. Belém-PA, 2010.

\begin{tabular}{|c|c|c|c|c|}
\hline \multirow[t]{2}{*}{ Variáveis } & \multicolumn{2}{|c|}{ ILPI } & \multicolumn{2}{|c|}{ Comunidade } \\
\hline & $\mathrm{m}$ & $d p$ & $\mathrm{~m}$ & $d p$ \\
\hline Idade & 77,5 & 10,4 & 72,2 & 6,1 \\
\hline Renda individual & 482,9 & 160,4 & 972,3 & 1756,4 \\
\hline Sexo & F & $\%$ & $\mathrm{~F}$ & $\%$ \\
\hline Feminino & 20 & 60,6 & 25 & 75,8 \\
\hline Masculino & 13 & 39,4 & 8 & 24,2 \\
\hline Escolaridade & $\mathrm{F}$ & $\%$ & F & $\%$ \\
\hline Analfabeto & 11 & 33,3 & 0 & 0 \\
\hline Até o fundamental & 18 & 54,6 & 29 & 87,9 \\
\hline Médio ou superior & 04 & 12,1 & 04 & 12,1 \\
\hline Presença de problemas de saúde & $\begin{array}{c}F \\
22\end{array}$ & $\begin{array}{c}\% \\
66,7\end{array}$ & $\begin{array}{c}F \\
25\end{array}$ & $\begin{array}{c}\% \\
75,8\end{array}$ \\
\hline Quantidade de amigos & $\mathrm{F}$ & $\%$ & F & $\%$ \\
\hline Nenhum ou poucos & 22 & 66,7 & 15 & 45,5 \\
\hline Muitos & 11 & 33,3 & 18 & 54,5 \\
\hline Recebe visitas & F & $\%$ & F & $\%$ \\
\hline Nunca ou raramente & 15 & 45,5 & 06 & 18,2 \\
\hline Frequentemente (semanal ou quinzenal) & 13 & 39,4 & 20 & 60,6 \\
\hline Recebe aposentadoria ou pensão & $\mathrm{F}$ & $\%$ & $\mathrm{~F}$ & $\%$ \\
\hline Sim & 31 & 94 & 28 & 87,5 \\
\hline Não & 02 & 06 & 04 & 12,5 \\
\hline Possui dependentes econômicos & $\mathrm{F}$ & $\%$ & $\mathrm{~F}$ & $\%$ \\
\hline $\operatorname{Sim}$ & 04 & 12,1 & 16 & 48,5 \\
\hline Não & 29 & 87,9 & 17 & 51,5 \\
\hline Quarto individual & $\mathrm{F}$ & $\%$ & $\mathrm{~F}$ & $\%$ \\
\hline Sim & 12 & 36,4 & 27 & 81,8 \\
\hline Não & 21 & 63,6 & 06 & 18,2 \\
\hline
\end{tabular}

$\mathrm{m}=$ média; $\mathrm{dp}=$ desvio-padrão; $\mathrm{F}=$ frequência; $\%=$ percentagem. 
No que diz respeito às percepções de controle e de qualidade de vida, foram encontradas diferenças significativas comparando-se idosos que viviam nas ILPIs e na comunidade (tabela 2). Os que viviam na comunidade possuíam melhor qualidade de vida (exceto na dimensão morte e morrer que não acusou diferença significativa entre os grupos) e maior percepção de controle primário com recursos próprios (CP1). Ressalta-se que todas as medianas do grupo de idosos residentes em ILPIs foram inferiores às medianas do grupo de idosos residentes na comunidade (tabela 2).

Tabela 2. Comparação entre idosos residentes na comunidade e em ILPIs - fatores da ECOPSE e do WHOQOL-OLD. Belém-PA, 2010.

\begin{tabular}{|c|c|c|c|c|c|c|}
\hline \multirow[t]{2}{*}{ Fatores avaliados } & \multicolumn{2}{|c|}{ Comunidade } & \multicolumn{2}{|c|}{ Institucionalizados } & \multirow{2}{*}{$\begin{array}{c}\text { Teste Mann- } \\
\text { Whitney }\end{array}$} & \multirow{2}{*}{$\begin{array}{c}\begin{array}{c}\text { Valor de } \mathrm{p}^{*} \\
\text { (Bicaudal) }\end{array} \\
\mathrm{p} \leq 0,05\end{array}$} \\
\hline & $\mathrm{md}$ & $\mathrm{m}$ postos & md & $\begin{array}{c}\mathrm{m} \\
\text { postos }\end{array}$ & & \\
\hline \multicolumn{7}{|l|}{$\begin{array}{l}\text { ECOPSE (Percepção } \\
\text { de controle) }\end{array}$} \\
\hline CP1 & 4,3 & 40,82 & 3,7 & 26,18 & 303,0 & 0,002 \\
\hline $\mathrm{CP} 2$ & 4,2 & 37,32 & 4,0 & 29,68 & 418,5 & 0,104 \\
\hline CS & 3,2 & 37,38 & 2,9 & 29,62 & 416,5 & 0,100 \\
\hline \multicolumn{7}{|l|}{$\begin{array}{l}\text { WHOQOL-OLD } \\
\text { (Qualidade de vida) }\end{array}$} \\
\hline FS & 4,3 & 39,14 & 3,8 & 27,86 & 358,5 & 0,017 \\
\hline AUT & 3,8 & 40,24 & 3,0 & 26,76 & 322,0 & 0,004 \\
\hline PPF & 3,8 & 39,05 & 3,5 & 27,95 & 361,5 & 0,018 \\
\hline PSO & 3,8 & 38,83 & 3,5 & 28,17 & 368,5 & 0,023 \\
\hline MEM & 4,5 & 33,83 & 4,3 & 33,17 & 533,5 & 0,886 \\
\hline INT & 3,8 & 39,52 & 2,8 & 27,48 & 346,0 & 0,011 \\
\hline GLOB & 3,8 & 40,48 & 3,3 & 26,52 & 314,0 & 0,003 \\
\hline
\end{tabular}

* Diferença significativa entre os dois grupos quando o valor de $\mathrm{p} \leq 0,05$.

$\mathrm{m}=$ média; $\mathrm{md}$ = mediana; $\mathrm{U}=$ resultado do teste Mann Whitney para verificar diferença entre as médias dos postos referentes aos valores de dois grupos; $\mathrm{p}=$ probabilidade de a hipótese nula ser verdadeira.

No que concerne às associações entre percepção de controle e qualidade de vida, foram encontradas as seguintes correlações, apresentadas por contexto:

Residentes em ILPIs: o fator "Controle primário com recursos próprios” (CP1) revelou correlação positiva com qualidade de vida, apenas na dimensão "Atividades Passadas Presentes e
Futuras" ( $\mathrm{p}=0,41 ; \mathrm{p}=0,02)$ do WHOQOL-OLD. Isto sugere que aqueles que se percebem com maiores possibilidades de produzir resultados desejados em seu ambiente são os mais satisfeitos com conquistas na vida e anseios. Os fatores "Controle primário com ajuda" (CP2) e "Controle secundário" (CS) não se correlacionaram de forma significativa com nenhum dos fatores do WHOQOL-OLD neste contexto. 
Moradores da comunidade: o fator "Controle primário com recursos próprios" (CP1) revelou correlação positiva com quase todas as dimensões do WOQOL-OLD, exceto "Participação Social e Intimidade": Funcionamento do Sensório $(\mathrm{p}=0,48 ; \mathrm{p}=0,01)$, Autonomia $(\mathrm{p}=0,49 ; \mathrm{p}=0,01)$, Atividades Passadas Presentes e Futuras $(\mathrm{p}=0,60$; $\mathrm{p}=0,001)$, Morte e Morrer $(\mathrm{p}=0,52 ; \mathrm{p}=0,01)$ e Fator Global de Qualidade de Vida ( $\mathrm{p}=0,65$; $\mathrm{p}=0,001)$. Este resultado sugere que aqueles que se percebem com maiores possibilidades de produzir resultados desejados em seu ambiente são os que têm melhor qualidade de vida, percebem menor impacto do envelhecimento sobre seu funcionamento sensorial, percebemse com maior liberdade para tomar as próprias decisões; mais satisfeitos com conquistas na vida e demonstram menor preocupação com a morte.

O fator "Controle primário com ajuda" (CP2) mostrou correlação positiva apenas com o fator "Morte e morrer" ( $\mathrm{p}=0,46 ; \mathrm{p}=0,01)$, indicando menor preocupação com a morte naqueles que pedem ajuda quando precisam para alcançar metas almejadas. O "controle secundário" (CS) não mostrou correlação significativa com nenhum dos fatores do WHOQOL-OLD neste contexto.

\section{DISCUSSÃO}

De acordo com os resultados encontrados, os idosos institucionalizados apresentaram percepção de controle primário (CP1) mais baixa, ou seja, revelaram menor confiança em sua própria capacidade para produzir resultados em seu ambiente do que aqueles que viviam na comunidade (tabela 2). Considerando-se que a percepção de controle está ligada à autonomia, um dos critérios da avaliação de qualidade de vida entre idosos, ${ }^{31}$ estes resultados oferecem apoio à literatura que aponta a institucionalização como fator negativo para a qualidade de vida, ${ }^{27,29}$ especialmente quando as instituições são carentes de recursos que possam prover variedade de atividades ${ }^{24}$ no sentido de estimular a percepção de controle e a qualidade de vida.
Este resultado era esperado, considerando-se a natureza da institucionalização, que por si só já reduz as possibilidades de exercer controle sobre a própria vida. $\mathrm{O}$ idoso institucionalizado fica impedido de gerir seus bens, tem restringida a liberdade de administrar seu tempo; seu espaço; relações sociais, e de tomar decisões. ${ }^{32}$ No contexto da institucionalização ocorrem perdas de referências; do sentimento de pertença, e a vontade individual fica submetida às decisões administrativas da instituição, podendo conduzir a apatia, passividade, alienação e anulação de identidade ${ }^{32}$ - portanto, à diminuição da percepção de controle. Além disso, observase que muitos idosos institucionalizados já são bem idosos, possuem saúde deteriorada, situação econômico-financeira precária e apresentam história de abandono ou descaso por parte dos familiares, quadro que os predispõe a baixa percepção de controle.

Diferentementedosidososinstitucionalizados, os idosos da comunidade têm mais possibilidade de exercer o poder de comando sobre suas próprias vidas, tomar decisões, fazer escolhas - enfim, possuem mais liberdade para exercer controle sobre o ambiente em que vivem.

Outro fator que pode afetar a percepção de controle nas instituições é o espaço pessoal disponível. Os idosos institucionalizados geralmente compartilham o quarto com outros idosos. No presente estudo, mais de 60\% dos residentes em ILPIs não tinham um quarto individual, contra apenas 18\% dos que viviam na comunidade (tabela 1). A elevada densidade social do ambiente de moradia é desfavorável à percepção de controle, ${ }^{33}$ uma vez que a privacidade fica comprometida. Isso é especialmente importante no caso do idoso institucionalizado, onde o único espaço realmente dele seria o próprio quarto.

As ILPIs se configuram como lares para idosos e têm a característica da coletividade, ou seja, em geral os idosos dividem o quarto com pelo menos mais uma pessoa. Há casos em que há seis pessoas em um quarto, fato que pode contribuir para dificultar a percepção 
de controle e a qualidade de vida dos idosos institucionalizados.

Ainda com relação à percepção de controle, vale ressaltar que, em ambos os grupos, o controle secundário teve valor baixo (mediana em torno do ponto médio da escala= 3) e inferior aos valores do controle primário (tanto CP1 quanto CP2) (tabela 2), indicando que, independentemente do contexto de existência, os idosos se percebem mais realizando esforços para alcançar suas metas diárias, do que se consolando por perdas. Isso corrobora achados de pesquisa ${ }^{9}$ realizada no Brasil que investigou crenças de controle em idosos.

Este é um resultado importante, especialmente tendo em vista que o critério de velhice saudável adotado pela Política Nacional de Saúde da Pessoa Idosa ${ }^{6}$ (PNSPI) é a manutenção da máxima capacidade funcional - independência e autonomia - pelo maior tempo possível. Nesse sentido, a crença no controle primário poderia favorecê-la. Por outro lado, na velhice, o escopo de ação no ambiente é reduzido, tanto pela perda de papéis sociais e suas consequências - tal como a diminuição das redes sociais - quanto pelos sinais de fragilidade que emergem em escala progressiva e contribuem para o surgimento de fatores limitantes como a diminuição da capacidade física, requerendo cada vez mais recursos de compensação.

Em meio a este quadro de perdas advindas com o avançar da idade, seria importante que os idosos desenvolvessem recursos pessoais de compensação psicológica e enfrentamento das perdas - controle secundário - no sentido de preservar seu potencial para o controle primário., ${ }^{917}$

Com relação à qualidade de vida, os resultados evidenciaram que os idosos institucionalizados obtiveram escores significativamente inferiores aos dos idosos residentes na comunidade em quase todas as dimensões avaliadas, exceto na dimensão "Morte e morrer" (tabela 2). Esses achados oferecem apoio aos estudos que compararam idosos da comunidade e institucionalizados, ${ }^{28,29}$ encontrando diferenças significativas com relação à qualidade de vida, com piores resultados para os institucionalizados. Além disso, demonstram que viver na comunidade favorece a qualidade de vida de idosos.

No entanto, apesar de a qualidade de vida percebida ser mais baixa entre os idosos institucionalizados, isto não quer dizer que seja ruim, já que a maioria das medianas girou em torno do ponto médio da escala (tabela 2), demonstrando um nível moderado de qualidade de vida, o que concorda com estudos que utilizaram o WHOQOL-OLD ${ }^{26,27}$ ou o WHOQOLbref ${ }^{25}$ com idosos institucionalizados.

Ainda com relação à qualidade de vida, o fator que obteve maior escore entre os institucionalizados foi "Morte e morrer" e os menores escores ficaram com os fatores "Autonomia" e "Intimidade". Esses resultados concordam com Dalsenter \& Matos, ${ }^{26}$ que encontraram menor escore para "Autonomia" e maior para "Morte e morrer", bem como com Nunes et al. ${ }^{27}$ que também obtiveram menor escore na faceta "Autonomia", o que ratifica a institucionalização como desfavorável ao poder de decisão - portanto ao exercício do controle.

No que diz respeito ao fator "Morte e morrer", cabe destacar que este foi o único em que os dois grupos avaliados não diferiram significativamente, indicando que, independentemente do contexto de existência, os idosos não estão preocupados com a morte - ou seja, não têm medo da morte e do que se relaciona a ela. Isso talvez se deva ao fato de que, sendo a velhice a última fase da vida e sabendo o idoso que a morte é a culminância desta fase, não adiantaria temê-la.

Uma explicação para isto poderia ser encontrada na Teoria da Dissonância Cognitiva, ${ }^{34}$ da Psicologia Social, segundo a qual, se duas cognições são dissonantes, elas geram um desconforto ao indivíduo que, então, procura restabelecer o equilíbrio psicológico por meio da mudança de uma delas. Nesse caso, por exemplo, a cognição "eu tenho medo da morte" é dissonante 
da cognição "minha morte é iminente". Como o idoso não pode modificar a cognição que diz que sua morte está próxima, ele restabeleceria o equilíbrio alterando a outra cognição, passando a encarar a morte com naturalidade, já que é um fato inevitável.

Quanto às associações entre percepção de controle e qualidade de vida, entre os idosos que viviam na comunidade, quatro das seis dimensões (FS, AUT, PPF e MEM) se correlacionaram positivamente com o controle primário (CP1). Os achados oferecem apoio a estudos que encontraram associação entre percepção de controle e indicadores de qualidade de vida entre idosos da comunidade, ${ }^{9,15,19,20-22}$ e estão de acordo com Haynes et al., ${ }^{13}$ que encontraram melhor percepção de bem-estar físico e psicológico entre idosos que utilizavam estratégias de controle primário para lidar com as restrições da idade.

Por outro lado, entre os idosos institucionalizados, somente uma (PPF) das seis dimensões de qualidade de vida avaliadas mostrou correlação positiva com o controle primário (CP1). Esta dimensão se refere à satisfação com conquistas na vida e coisas a que se anseia ${ }^{31}$ (ex. de itens: Quão satisfeito você está com aquilo que alcançou na sua vida? Até que ponto você está satisfeito com as suas oportunidades para continuar alcançando outras realizações na sua vida?). Como o controle primário com recursos próprios se refere à percepção de ser capaz de produzir os resultados desejados em seu ambiente, ou seja, o senso de estar no comando da sua vida ${ }^{10}$ (ex. de itens: Quando realmente quero alguma coisa, faço todo o possível para conseguir. Quando planejo fazer alguma coisa, acredito firmemente que vou conseguir realizar), pode-se argumentar, por um lado, que aqueles que têm maior controle sobre seu ambiente (p.ex., saem mais da instituição, têm um quarto só para si, administram uma parte maior de seu próprio dinheiro), percebem melhor qualidade de vida neste aspecto, ou seja, se percebem mais satisfeitos com suas conquistas e com maiores possibilidades de novas realizações. Por outro lado, a percepção de satisfação com o que se alcançou na vida pode estar contribuindo para melhorar o senso de controle entre esses idosos. Assim, argumenta-se que atividades que possibilitem o resgate de elementos da identidade do idoso que fazem parte de sua história passada e que apontam para realizações/conquistas, podem favorecer o senso de controle e contribuir para a qualidade de vida dentro da instituição.

Este estudo apresenta algumas lacunas (limitações) a serem preenchidas em investigações futuras do tema. O questionário sociodemográfico, embora longo, não abordou algumas questões cujas respostas poderiam ter auxiliadoa compreenderos resultados desteestudo - por exemplo, a presença/ausência de cuidadores privados entre os idosos institucionalizados, bem como o grau de dependência que tinham do cuidador institucional. Acredita-se que isto possa ter ocorrido em função de este estudo ter sido um recorte de pesquisa mais ampla que envolveu idosos da comunidade e institucionalizados, com vistas a estudar diversos fatores psicossociais, inclusive percepção de controle e qualidade de vida. Assim, o protocolo de pesquisa ficou extenso e alguns aspectos específicos acerca de dados sociodemográficos, embora importantes, não foram abordados.

Outro aspecto a ser mencionado é que as informações acerca das condições cognitivas do idoso para atender ao protocolo da pesquisa foram obtidas verbalmente junto a profissionais como psicólogos e assistentes sociais, ou inferidas a partir do contato inicial com o idoso. Isto poderia ser realizado de forma mais precisa, em investigações futuras, por meio de instrumentos confiáveis de rastreio para capacidade cognitiva.

\section{CONCLUSÃO}

Os resultados encontrados permitem concluir que viver na comunidade favorece tanto a percepção de controle, quanto a qualidade de vida, em comparação a viver nas instituições de longa permanência para idosos. Os dados permitem concluir também que viver na comunidade favorece a associação entre percepção de controle primário e qualidade de 
vida, uma vez que esta associação se fez presente em maior número de domínios de qualidade de vida nos idosos que vivem na comunidade, comparados aos institucionalizados.

Considera-se que os objetivos propostos foram alcançados e as perguntas de pesquisa respondidas. Espera-se ter contribuído para o panorama dos estudos sobre qualidade de vida entre idosos institucionalizados, comparandoos com idosos que vivem na comunidade, apresentando uma amostra na Região Norte do Brasil. Espera-se também ter fornecido subsídios para a compreensão da relação entre qualidade de vida e percepção de controle, enquanto aspecto psicossocial importante para a velhice saudável.

\section{REFERÊNCIAS}

1. De Luca DE, Bonacci S, Giraldi G. Aging populations: the health and quality of life of the elderly. Clin Ter 2011;162(1):13-8.

2. Falcade BL, Leite MT, Hildebrandt LM, Kirchner RM, Silva LAA, Gonçalves LHT. Octogenários em residências unipessoais: enfoque sobre a qualidade de vida e condições de saúde. Rev Enferm UFSM 2011;1(3):386-93.

3. Theofilou P. Quality of Life: definition and measurement. Europ J Psychol 2013;9(1):150-62.

4. Fleck MPA, organizador. A avaliação de qualidade de vida: guia para profissionais da saúde. Porto Alegre: Artmed; 2008.

5. World Health Organization. Functional decline and dependence in ageing populations. In: 66th World Health Assembly - Promoting Health through the life-course; 2013 May 20-28; Geneva. Geneva: WHO; 2013.

6. Brasil. Portaria No. 2528 de 19 de outubro de 2006. Aprova a Política Nacional de Saúde da Pessoa Idosa. Brasília: Ministério da Saúde; 2006.

7. Moraes EM. Avaliação multidimensional do idoso: instrumentos de rastreio. 2. ed. Belo Horizonte: Folium; 2009.

8. Neri AL. Crenças de auto-eficácia e envelhecimento bem-sucedido. In: Freitas EV, Py L, Cançado FAX, Doll J, Gorzoni ML. Tratado de geriatria e gerontologia. $2^{a}$ ed. Rio de Janeiro: GuanabaraKoogan; 2006. p. 1267-76.

9. Khoury HTT. Controle primário e controle secundário: relação com indicadores de envelhecimento bem-sucedido [tese]. Brasília, DF: Universidade de Brasília; 2005.

10. Khoury HTT, Günther IA. Desenvolvimento de uma medida de controle primário e secundário para idosos. Psicol Teor Pesqui 2013;29(3):277-85.

11. Resende MC, Rabelo DF. Senso de controle e saúde no envelhecimento. In: Freitas EV, Py L, Cançado FAX, Doll J, Gorzoni ML. Tratado de geriatria e gerontologia. 3. ed. Rio de Janeiro: Guanabara Koogan; 2013. p.1547-52.

12. Chipperfield JG, Newall NE, Perry RP, Stewart TL, Bailis DS, Ruthig JC. Sense of control in late life: Health and survival implications. Pers Soc Psychol Bull 2012;38(8):1081-92.

13. Haynes TL, Heckhausen J, Chipperfield JG, Perry RP, Newall NE. Primary and secondary control strategies: implications for health and well-being among older adults. J Soc Clin Psychol 2009;28:165-97.

14. Stocks A, April KA, Lynton N. Locus of control and subjective well-being: a cross cultural study. Probl perspect manag 2012;10(1):17-25.

15. Lachman ME, Agrigoroaei S. Promoting functional health in midlife and old age: long-term protective effects of control Beliefs, social support, and physical exercise. Plos One [Internet] 2010 [acesso em 24 abril 2014];5(10):1-9. Disponível em http://www.ncbi.nlm. nih.gov/pmc/articles/PMC2952603/

16. Mardiyono MNS, Praneed S, Wongchan P. Concept Analysis of Perceived Control. Nurse Media J Nurs 2011;1(2):225-43.

17. Rothbaum F, Weisz JR, Snyder SS. Changing the world and changing the self: a two-process model of perceived control. J Pers Soc Psychol 1982;42(1):5-37.

18. Khoury HTT, Günther IA. Processos de autoregulação no curso de vida: controle primário e controle secundário. Psicol Reflex Crít 2009;22(1):144-52.

19. Ferguson SJ, Goodwin AD. Optimism and WellBeing in Older Adults: the mediating role of social support and perceived control. Int J Aging Hum Dev 2010;71(1):43-68. 
20. Bowling A, Seetai S, Morris R, Ebrahim S. Quality of life among older people with poor functioning. The influence of perceived control over life. Age Ageing 2007;36:310-15.

21. Oliveira LS, Rabelo, DF, Queroz NC. Estilo de vida, senso de controle e qualidade de vida: um estudo com a população idosa de Patos de Minas-MG. Estud Pesqui Psicol 2012;12(2),416-30.

22. Timm L A. A qualidade de vida no idoso e sua relação com o locus de controle [Dissertação]. Porto Alegre: PUC/RGS; 2006.

23. Camarano A A, Kanso S. As instituições de longa permanência para idosos no Brasil. Rev Bras Est Pop 2010;27(1):233-35.

24. Bradshaw SA, Playford ED, Riazi A. Living well in care homes: a systematic review of qualitative studies. Age Ageing 2012;41:429-40.

25. Lima DL, Lima MAVD, Ribeiro CG. Envelhecimento e qualidade de vida de idosos institucionalizados. Rev Bras Ciênc Envelhec Hum 2010;7(3):346-56.

26. Dalsenter CA, Matos FM. Percepção da qualidade de vida em idosos institucionalizados da cidade de Blumenau (SC). Dynamis Rev Tecnocientífica 2009;15(2):32-7.

27. Nunes VMA, Menezes RMP, Alchieri JC. Avaliação da qualidade de vida em idosos institucionalizados no município de Natal, Estado do Rio Grande do Norte. Acta Sci., Health Sci 2010;32(2):119-26.
28. Vitorino LM, Paskulin LMG, Vianna LAC. Qualidade de vida de idosos da comunidade e de instituições de longa permanência: estudo comparativo. Rev Latinoam Enferm 2013;21:1-9.

29. Oliveira ERA, Gomes MJ, Paiva KM. Institucionalização e qualidade de vida de idosos da região metropolitana de Vitória - ES. Esc Anna Nery Enferm 2011;15(3):518-23.

30. Dias DSG, Carvalho CS, Araújo CV. Comparação da percepção subjetiva de qualidade de vida e bemestar de idosos que vivem sozinhos, com a família e institucionalizados. Rev Bras Geriatr Gerontol 2013;16(1):127-38.

31. Organização Mundial da Saúde; Power M, Schmidt S. WHOQOL-OLD Manual [Internet]. Chachamovich E, Fleck MP, adaptadores. [S.1.: s.n.], [200-] [acesso em 31 abril 2014]. Disponível em http://www. ufrgs.br/psiquiatria/psiq/WHOQOL- OLD\%20 Manual\%20POrtugues.pdf

32. Herédia VBM, Casara MB, Cortelletti IA, Ramalho $\mathrm{MH}$, Sassi A. A realidade do idoso institucionalizado. Textos Envelhecimento 2004;7(2):1-12.

33. Khoury HTT, Günther IA. Ambiente de moradia e controle primário em idosos. Paidéia 2008;18(39):53-60.

34. Festinger L. Teoria da dissonância cognitiva. São Paulo: Cortez; 1977. 